\title{
Breakdown of the nuclear-spin-temperature approach in quantum-dot demagnetization experiments
}

\section{P. Maletinsky $\star$, M. Kroner and A. Imamoglu}

The physics of interacting nuclear spins arranged on a crystalline lattice is generally described using a thermodynamic framework ${ }^{1}$ and the concept of spin temperature. In the past, experimental studies in bulk solid-state systems have proven this concept to be not only correct $^{2,3}$ but also vital for the understanding of experimental observations ${ }^{4}$. Here we show, using demagnetization experiments, that the concept of spin temperature in general fails to describe the mesoscopic nuclear-spin ensemble of a quantum dot. We associate the observed deviations from a thermal spin state with the presence of strong quadrupolar interactions within the quantum dot, which cause significant anharmonicity in the spectrum of the nuclear spins. Strain-induced, inhomogeneous quadrupolar shifts also lead to a complete suppression of angularmomentum exchange between the nuclear-spin ensemble and its environment, resulting in nuclear-spin relaxation times exceeding an hour. Remarkably, the position-dependent axes of the quadrupolar interactions render magnetic-field sweeps inherently non-adiabatic, thereby causing an irreversible loss of nuclear-spin polarization.

The study of nuclear-spin physics by optical orientation experiments in bulk semiconductor materials has been an active field of research over recent decades ${ }^{5-7}$. These research efforts have shown that, using the electron as a mediator, it is possible to transfer angular momentum from light onto nuclei, thereby establishing a nuclear-spin polarization that is orders of magnitude higher than the equilibrium nuclear polarization at cryogenic temperatures. As a result, the effective nuclear-spin temperature in such an optically pumped system can be pushed to the microkelvin regime. Combining these optical pumping schemes with nuclear adiabatic demagnetization techniques borrowed from bulk nuclear magnetic resonance experiments ${ }^{3}$ would be a natural extension to these experiments that could lead to a significant further reduction of the nuclear-spin temperature. This approach, previously demonstrated in bulk semiconductors ${ }^{5,8}$, suffers from the fact that, in most systems where optical orientation of nuclear spins is possible, nuclear-spin relaxation is too fast to allow for a significant reduction of magnetic fields in an adiabatic way. Here, we use the exceedingly long nuclear-spin relaxation time in self-assembled quantum dots (QDs) (ref. 9) to implement an 'adiabatic' demagnetization experiment on the system of $\sim 10^{5}$ nuclear spins.

The mesoscopic ensemble of nuclear spins in a QD can be conveniently polarized and measured by optical means $s^{5,9-12}$. To this end, we use the photoluminescence of the negatively charged exciton $\left(X^{-1}\right)$ under resonant excitation of an excited QD state. It has been shown previously ${ }^{13}$ that, under appropriate excitation conditions, $20-50 \%$ of the QD nuclear spins can be efficiently a

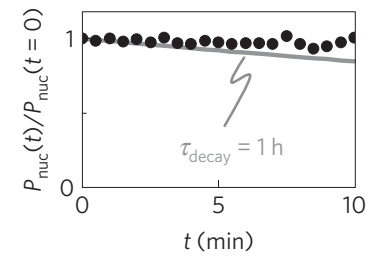

b

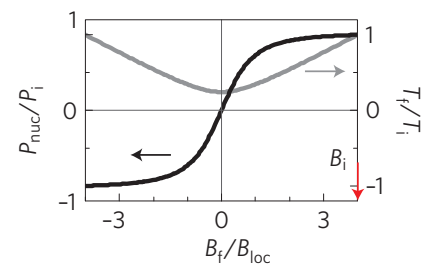

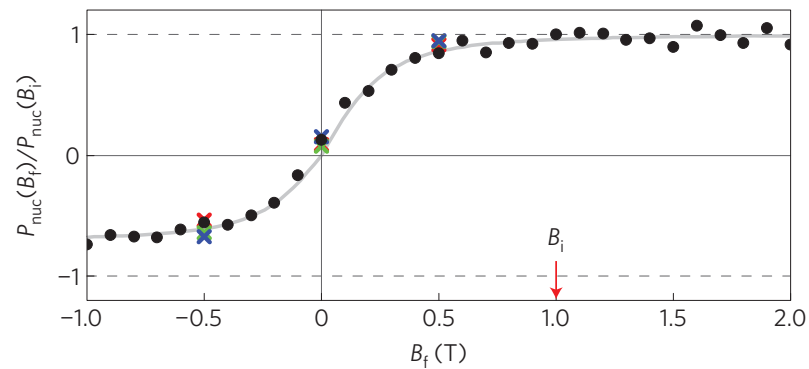

Figure 1 | Demagnetization of QD nuclear spins. a, Free decay of $P_{\text {nuc }}$ at $B_{\text {ext }}=2 \mathrm{~T}$ for an uncharged QD after optical pumping of the nuclear spins for $\tau_{\text {pump }}=600 \mathrm{~ms}$. The grey curve shows an exponential decay with a time constant of $1 \mathrm{~h}$ for comparison. $\mathbf{b}$, Theoretical prediction of nuclear-spin temperature and polarization during adiabatic demagnetization from a field $B_{\mathrm{i}}$ (red arrow) to $B_{\mathrm{f}}$. C, Schematic diagram of the experimental procedure for adiabatic demagnetization of QD nuclear spins. The nuclei are optically pumped at $B_{\text {ext }}=B_{\mathrm{i}}\left(T_{\mathrm{spin}, i} \sim \mathrm{mK}\right)$. Directly after the pumping pulse, the electron is ejected from the QD. $B_{\text {ext }}$ is then linearly ramped at a rate $\gamma_{B}$ to a value $B_{\mathrm{f}}$, at which we measure $P_{\text {nuc }}$. $\mathbf{d}$, Experimental (de)magnetization of QD nuclear spins. $B_{\mathrm{i}}=1 \mathrm{~T}$ as indicated by the red arrow, $\gamma_{B}=10 \mathrm{mT} \mathrm{s}^{-1}$ and $\Delta E_{\mathrm{OS}}\left(B_{\mathrm{i}}\right)=57 \mu \mathrm{eV}$. The grey curve is a fit according to the theoretical predictions shown in $\mathbf{b}$; we find $B_{\text {loc }}=290 \mathrm{mT}$. Blue, green and red crosses show a similar experiment, with $\gamma_{B}=5,2.5$ and $0.8 \mathrm{mT} \mathrm{s}^{-1}$, respectively ( $B_{\mathrm{i}}=0.5 \mathrm{~T}$ for these data points).

polarized in a timescale of a few milliseconds. The resulting dynamical nuclear-spin polarization can then be measured through a change in the Zeeman splitting, $\Delta E_{\mathrm{OS}}$, of the $X^{-1}$ recombination line ${ }^{13}$; this energy shift due to the spin-polarized nuclei is commonly referred to as the Overhauser shift ${ }^{14}$. 
A remarkable feature of the QD nuclear-spin system is the excellent isolation from its environment if the QD is uncharged. Figure 1a shows the corresponding free evolution of the nuclearspin polarization $P_{\text {nuc }}$ (proportional to $\Delta E_{\mathrm{OS}}$ ) in a QD subject to an external magnetic field $B_{\text {ext }}=2 \mathrm{~T}$. The nuclear-spin relaxation time clearly exceeds one hour and does not vary appreciably over the magnetic-field range relevant to this work ${ }^{9}$. As the bulk material surrounding the QD remains unpolarized during the experiment (see the Methods section), the long nuclear-spin lifetime indicates that nuclear-spin diffusion between the QD and its environment is strongly suppressed. We attribute this quenching of spin diffusion to the structural and chemical mismatch between the InGaAs QD and its GaAs surroundings ${ }^{12,15}$. The very slow nuclear-spin relaxation leaves room for further manipulation of the QD nuclearspin system after optical pumping. In particular, we can study how $P_{\text {nuc }}$ behaves under slow variations of external parameters and thereby study the validity of spin thermodynamics for the QD nuclear-spin system.

If the QD nuclei were describable using a thermodynamic approach, $P_{\text {nuc }}$ would be aligned with $B_{\text {ext }}$ and would be described by Curie's law $\gamma P_{\text {nuc }}=B_{\text {ext }} C / T_{\text {spin }}$ (ref. 3) (here, $\gamma$ is the nuclear gyromagnetic ratio, $C$ the Curie constant and $T_{\text {spin }}$ the nuclear-spin temperature). An adiabatic lowering of $B_{\text {ext }}$ from an initial value $B_{\mathrm{i}}$ to a final value $B_{\mathrm{f}}$ would conserve $P_{\text {nuc }}$ and lead to a reduction of $T_{\text {spin }}$ by a factor $B_{\mathrm{f}} / B_{\mathrm{i}}$. In general, cooling by adiabatic demagnetization is possible for any system where the spin entropy $S$ is conserved and a function of $B_{\text {ext }} / T_{\text {spin }}$ only. The ultimate limit to the achievable cooling is determined by nuclear-spin interactions, which give the dominant contribution to $S$ at low magnetic fields. The strength and nature of these interactions can be phenomenologically described by a random local magnetic field $B_{\text {loc }}$. In most cases, $B_{\text {loc }}$ is given by the nuclear dipolar couplings $(\approx 0.1 \mathrm{mT})$. As soon as $B_{\text {ext }} \approx B_{\text {loc }}$, the local fields randomize an established nuclear-spin polarization and thereby limit the efficiency of the adiabatic spin cooling to $B_{\mathrm{loc}} / B_{\mathrm{i}}$. The resulting behaviour of nuclear-spin temperature and polarization as a function of $B_{\mathrm{f}}$ is sketched in Fig. $1 \mathrm{~b}$ : for $B_{\text {ext }}=0$, the spin temperature remains finite and the nuclear spins are completely depolarized. Amazingly, this depolarization is a reversible process, provided that $S$ is a conserved quantity at all fields. When the spins are re-magnetized to a magnetic field exceeding $B_{\text {loc }}$, their polarization recovers along the direction of the magnetic field and in particular conserves the sign of its initial spin temperature.

To test the validity of spin thermodynamics for the QD nuclear spins and to study the possibility of adiabatic cooling in this system, we performed demagnetization experiments on a $\mathrm{QD}$, as illustrated in Fig. 1c. A circularly polarized 'pump' pulse of length $\tau_{\text {pump }}$ is used to polarize the nuclear spins. After ejecting the electron from the $\mathrm{QD}$, we linearly ramp $B_{\text {ext }}$ from $B_{\mathrm{i}}$ to $B_{\mathrm{f}}$ with a rate $\gamma_{\mathrm{B}}=10 \mathrm{mT} \mathrm{s}^{-1}$. At the final field $B_{\mathrm{f}}$, the remaining degree of nuclear-spin polarization is measured using a linearly polarized 'probe' pulse of length $\tau_{\text {probe }}$ (ref. 9). This experiment is repeated at various values of $B_{\mathrm{f}}$ to record the process of 'adiabatic' (de)magnetization.

Figure 1d shows the result of a demagnetization experiment performed on the nuclear-spin system of an individual QD. The nuclei are polarized with a pump pulse $\tau_{\text {pump }}=300 \mathrm{~ms}$ at $B_{\mathrm{i}}=1 \mathrm{~T}$ and measured at $B_{\mathrm{f}}$ with a probe pulse $\tau_{\text {probe }}=5 \mathrm{~ms}$. At a rough glance, this measurement qualitatively follows the behaviour depicted in Fig. 1b. A closer inspection, however, reveals significant deviations: on ramping the external field to $B_{\mathrm{f}}=-1 \mathrm{~T}$ we recover only $63 \%$ of the initial $P_{\text {nuc }}$. In addition, by measuring $P_{\text {nuc }}\left(B_{\mathrm{f}}\right)$ we determined the value of the local field to be $B_{\text {loc }}=290 \mathrm{mT}$ : this value is about three orders of magnitude larger than typical nuclear dipolar fields. Finally, we observe that even for $B_{\mathrm{f}}=0$ the QD has a remanent nuclear-spin polarization $P_{\text {nuc }}^{\text {rem }}$. To verify that we do not induce an unwanted increase of spin entropy by sweeping $B_{\text {ext }}$
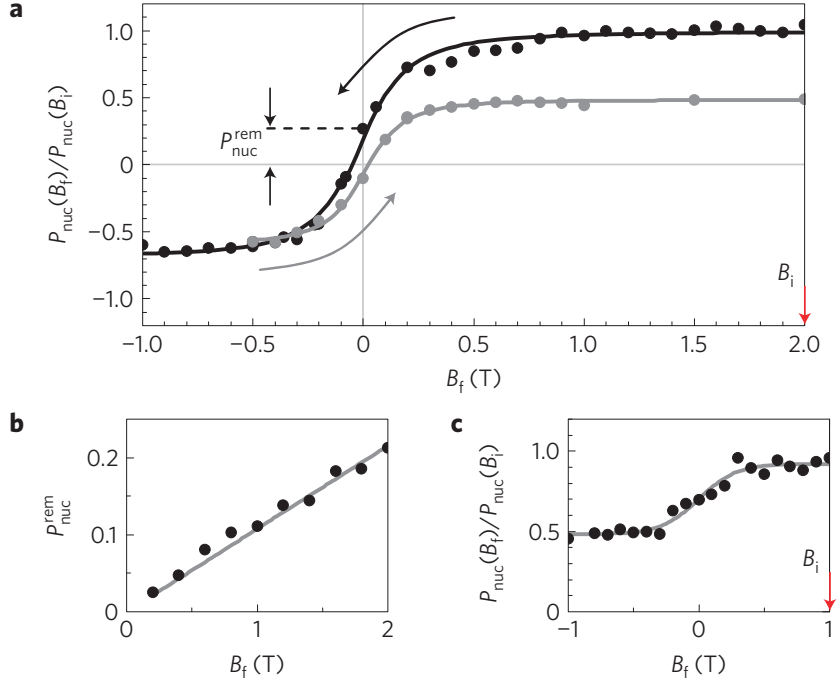

Figure 2 | Irreversibility and hysteresis in the demagnetization experiment. a, Black circles, the same experiment as in Fig. 1d, with $B_{\mathrm{i}}=2.0 \mathrm{~T}$ as indicated by the red arrow $\left(\Delta E_{\mathrm{OS}}\left(B_{\mathrm{i}}\right)=89.5 \mu \mathrm{eV}\right)$. After reaching $B_{\mathrm{f}}^{\prime}=-1 \mathrm{~T}$, we reverse the magnetic-field sweep direction and bring the nuclei back to the initial field (grey circles). b. The remanent nuclear-spin polarization $P_{\text {nuc }}^{\text {rem }}$ (normalized to the value $P_{\text {nuc }}\left(B_{i}\right)$ found in a) as a function of $B_{\mathrm{i}}$. As $P_{\text {nuc }}\left(B_{\mathrm{i}}\right) \propto B_{\mathrm{i}}$, the nuclear-spin temperature after optical pumping is roughly constant for all values of $B_{i}$ in this measurement. c, After polarization of nuclear spins at $B_{\mathrm{i}}=1 \mathrm{~T}$ (red arrow), we sweep $B_{\text {ext }}$ to $B_{f}^{\prime}$ and then back to $B_{\mathrm{i}}$, where $P_{\text {nuc }}$ is measured. The magnetic field sweeps become partly irreversible as soon as $\left|B_{f}^{\prime}\right| \lesssim 0.3 T \approx B_{Q}$. The lines in the figures are guides to the eye.

too fast, we repeated our experiment for values of $\gamma_{\mathrm{B}}$ of $5,2.5$ and $0.8 \mathrm{mT} \mathrm{s}^{-1}$ (crosses in Fig. 1d). Within the experimentally accessible range, $\gamma_{\mathrm{B}}$ has no influence on our observations.

The discrepancy between our experimental findings and the predictions from a thermodynamical treatment of nuclear spins becomes even more pronounced if we increase $P_{\text {nuc }}\left(B_{\mathrm{i}}\right)$ (which can be achieved by first increasing $B_{\text {ext }}$ to $2.2 \mathrm{~T}$ (refs 12,16$)$ ). Figure $2 \mathrm{a}$ shows an experiment where we then demagnetize the polarized nuclear spins starting from $B_{\mathrm{i}}=2.0 \mathrm{~T}$ to a final field $B_{\mathrm{f}}^{\prime}=-1 \mathrm{~T}$ (black data points). We then reverse the sweep direction of the magnetic field and ramp $B_{\text {ext }}$ back to $B_{\mathrm{f}}$ (grey data points). This experiment shows a considerable hysteresis of the nuclear-spin polarization as a function of $B_{\mathrm{f}}$. In particular, $P_{\text {nuc }}^{\mathrm{rem}}$ changes sign for the two sweep directions of $B_{\text {ext }}$. Furthermore, the magnitude of $P_{\text {nuc }}^{\text {rem }}$, and respectively the width of the observed hysteresis curve, depends linearly on the initial degree of nuclear-spin polarization and on $B_{\mathrm{i}}$ (Fig. 2b).

To obtain more information about the source of irreversibility of $P_{\text {nuc }}$ during magnetic-field sweeps, we performed a further experiment, where we optically orient the nuclear spins at $B_{\mathrm{i}}=1 \mathrm{~T}$ and ramp the field to a value $B_{\mathrm{f}}^{\prime}<B_{\mathrm{i}}$ and then back to $B_{\mathrm{i}}=B_{\mathrm{f}}$, where we measure the remaining degree of nuclear-spin polarization. The result of this experiment (Fig. 2c) indicates that the magneticfield sweeps start to induce irreversibilities in $P_{\text {nuc }}$ as soon as $\left|B_{\text {ext }}\right| \lesssim B_{\text {loc }} \approx 300 \mathrm{mT}$.

Finally we note that the experimental observations described here do not depend on the sign of the initial nuclear-spin temperature $\left(T_{\text {spin, } i}\right)$. We have repeated the demagnetization experiments for $T_{\text {spin, } i}<0$ (that is, $\sigma^{-}$laser excitation at $B_{\mathrm{i}}>0$, not shown here) and observed values of $B_{\text {loc }}$ and $P_{\text {nuc }}^{\text {rem }}$ consistent with the measurements presented in Figs 1 and 2. These measurements are complicated by the fact that for $T_{\text {spin }}<0$ nuclear-spin pumping is rather inefficient ${ }^{12}$, leading to a low degree of dynamical 
nuclear-spin polarization and therefore a smaller signal-to-noise ratio than for $T_{\text {spin }}>0$.

The three principal features of our experiments, the existence of $P_{\text {nuc }}^{\text {rem }}$, the hysteretic behaviour of $P_{\text {nuc }}$ and the partial irreversibility of our demagnetization experiment, result from a violation of the nuclear (Zeeman) spin temperature approximation ${ }^{1,3}$. We explain these features by taking into account the strong inhomogeneous quadrupolar interaction (QI) of the nuclear spins in a QD (refs 17-19). The self-assembled growth of InGaAs QDs is driven by a strong lattice mismatch between InGaAs and its surrounding GaAs matrix, which results in a heavily strained QD lattice. As a consequence, QD nuclei experience large electric-field gradients, which couple to the nuclear quadrupolar moment. The resulting quadrupolar Hamiltonian $^{20}$,

$$
\hat{H}_{\mathrm{Q}}=\frac{h v_{\mathrm{Q}}}{2}\left(\hat{I}_{z^{\prime}}^{2}-\frac{1}{3} I(I+1)\right)
$$

is characterized by a nuclear quadrupolar frequency $v_{\mathrm{Q}}$ (proportional to the local strain at the nuclear site) and a quadrupolar axis $z^{\prime}$ (with corresponding unit vector $\mathbf{e}_{z^{\prime}}$ along the main axis of the local electric-field-gradient tensor). $\hat{\mathbf{I}}$ is the nuclear-spin angularmomentum operator with quantum number $I$ and $\hat{I}_{z^{\prime}}=\hat{\mathbf{I}} \cdot \mathbf{e}_{z^{\prime}}$. For typical strain values of $2 \%$ (ref. 21 ), we find $v_{\mathrm{Q}} \approx 2.8 \mathrm{MHz}$ for As and 1.2 $\mathrm{MHz}$ for In (ref. 22). For comparison of the interaction strength of $\hat{H}_{\mathrm{Q}}$ with a pure nuclear Zeeman Hamiltonian $\hat{H}_{\mathrm{Z}}=\gamma \hat{\mathbf{I}} \cdot \mathbf{B}_{\text {ext }}$, it is convenient to express the QI strength by an equivalent magnetic field $B_{\mathrm{Q}}=h \nu_{\mathrm{Q}} / \gamma$. For As and In, we find $B_{\mathrm{Q}}=388 \mathrm{mT}$ and $125 \mathrm{mT}$, respectively; the corresponding mean value agrees well with our experimental estimate for $B_{\text {loc }}$.

The spectrum of a nuclear spin with quadrupolar frequency $v_{\mathrm{Q}}$ depends strongly on the angle $\theta$ between $\mathbf{e}_{z^{\prime}}$ and the external magnetic field (directed along $\mathbf{e}_{z}$, Fig. 3a). Figure $3 \mathrm{~b}$ shows the eigenenergies of a nuclear spin with $I=3 / 2$, as a function of $B_{\text {ext }} / B_{\mathrm{Q}}$. At $B_{\text {ext }}=0$, the spectrum is governed by $\hat{H}_{\mathrm{Q}}$, which pairs the nuclear-spin states into doublets with angular-momentum projections $\pm m_{z^{\prime}}$ on $\mathbf{e}_{z^{\prime}}$. The doublets are split by an energy $\left|\hbar \omega_{m_{z^{\prime}}, m_{z^{\prime}}+1}\right|=\left(m_{z^{\prime}}+1 / 2\right) h v_{\mathrm{Q}}$, respectively. Conversely, in a high magnetic field, the spectrum is determined by $\hat{H}_{\mathrm{Z}}$ with nuclear angular momentum being quantized along the axis $\mathbf{e}_{z}$. Even at arbitrarily high fields, however, the spectrum is significantly perturbed by $\hat{H}_{\mathrm{Q}}$ and never becomes perfectly harmonic.

We modelled our demagnetization experiment using the steadystate solution of a rate equation for the populations $p_{|m\rangle}$ of spin states $|m\rangle$, which are mutually coupled through dipolar interactions (Fig. $3 \mathrm{~b}$ and $\mathrm{c}$ ). The nuclear spins are initialized with a Boltzmann distribution at $B_{\text {ext }}=B_{\mathrm{i}}$ (see the Methods section) and the evolution of the $p_{|m\rangle}$ is calculated as a function of $B_{\text {ext }}$. Owing to the unequal nuclear-spin level spacings, only nuclear-spin flip-flops that preserve $p_{|m\rangle}(\forall|m\rangle)$ are energetically allowed in general and therefore the spin populations remain invariant as a function of $B_{\text {ext }}$. Varying $B_{\text {ext }}$ will change the relative nuclear-spin level spacings in the nonlinear way depicted in Fig. 3c. As the $p_{|m\rangle}$ remain invariant while $B_{\text {ext }}$ is reduced, the nuclear spins are driven into a state that is out of thermal equilibrium (that is, not Boltzmann distributed). At specific values of $B_{\text {ext }}$ (red markers in Fig. 3c), transition energies between distinct pairs of nuclear-spin states can coincide-a situation denoted as a 'crossover' of nuclear-spin transitions ${ }^{1}$. At these fields, the $p_{|m\rangle}$ are no longer constant and the nuclear-spin levels involved in the crossover can relax to a Boltzmann distribution. The irreversibility observed in our magnetic-field sweeps is a consequence of this partial relaxation of nuclear spins to thermal equilibrium. We speculate that the resulting increase of the nuclearspin entropy is induced by an energy-conserving coupling to the a

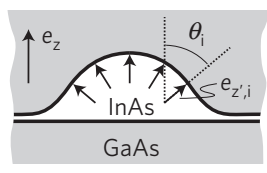

b

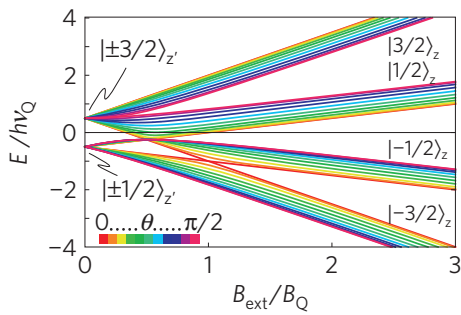

c

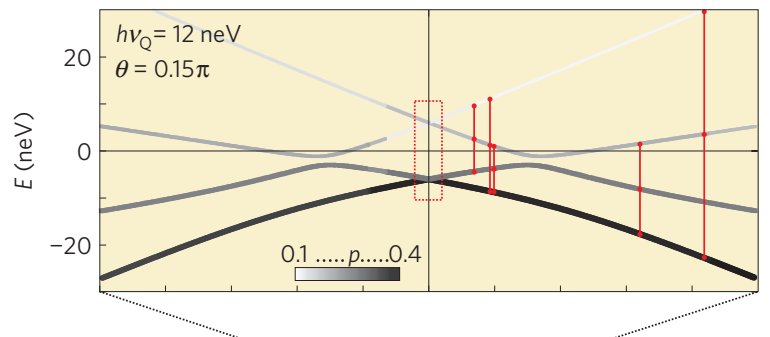

d

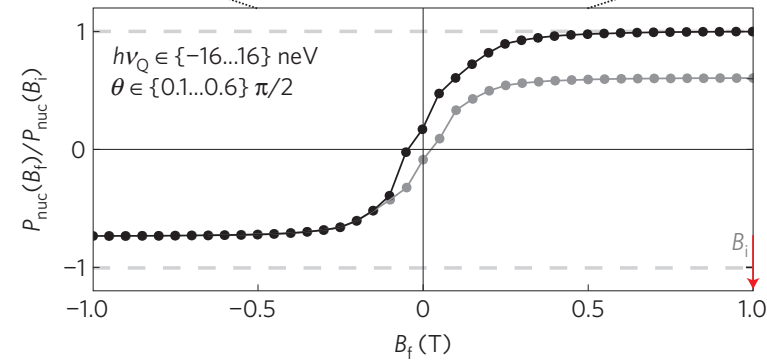

Figure 3 | Modelling of the demagnetization experiment. Local electric-field gradients induced by strain in self-assembled QDs result in strong QI for the nuclear spins. a, Model of local strain axis distribution $\mathbf{e}_{z^{\prime}}$ within a QD. b. Spectrum of nuclear spins $(I=3 / 2)$ under the influence of both $\hat{H}_{\mathrm{Q}}$ and $\hat{H}_{\mathrm{Z}}$ for a variety of angles $\theta$ between $\mathbf{e}_{z^{\prime}}$ and $\mathbf{e}_{\mathrm{z}}$. c. Simulation of QD nuclear-spin demagnetization for a particular setting $v_{\mathrm{Q}}=3 \mathrm{MHz}$ and $\theta=0.15 \pi$. Nuclear-spin populations $p$ are represented both by line thickness and greyscale of the lines that indicate the energy of the nuclear-spin states. At $B_{\mathrm{i}}=1 \mathrm{~T}$, the nuclei are initialized with a Boltzmann distribution over their spin states. The populations remain constant for most values of $B_{f}$. Only if a crossover of nuclear-spin transitions occurs (red markers for $B_{f}>0$ ) do the occupations of the involved spin states evolve to a (local) thermal equilibrium distribution (see the text). We simulate this process for a set of configurations $\left\{\theta, v_{\mathrm{Q}}\right\}$ and calculate the corresponding magnetization $P_{\text {nuc }} \propto\left\langle I_{z}\right\rangle$. d, The resulting nuclear-spin polarization as a function of $B_{f}$ starting at $B_{i}$ (red arrow), which qualitatively reproduces the experimental findings shown in Fig. 2.

environment of the nuclear spins. If the minimal energy gap of the anticrossing induced by the dipolar coupling between two interacting nuclear spins at their crossover is smaller than the coupling to the environment, pure dephasing of the nuclearspin transitions will induce irreversible crossover transitions and $S$ will increase.

On sweeping $B_{\text {ext }}$ through zero (red box in Fig. 3c), dipolar interactions will couple the states $m_{z^{\prime}}= \pm 1 / 2$. The associated passage through the avoided crossing between these single-spin states is adiabatic and preserves the respective populations in the two lowest-lying spin states. In contrast, nuclear dipolar interaction cannot couple any of the states with $\left|m_{z^{\prime}}\right|>1 / 2$ owing to conservation of energy and angular momentum. The spin states in the $\left|m_{z^{\prime}}\right|=3 / 2$ manifold will therefore cross and in particular preserve their populations $p_{3 / 2}$ and $p_{-3 / 2}$. The imbalance between these populations $\left(p_{3 / 2}<p_{-3 / 2}\right.$ in Fig. 3$)$ will result in a remnant polarization $P_{\text {nuc }}^{\text {rem }}$, even if $B_{\text {ext }}$ is strictly zero.

We averaged our model over a set of parameters $\theta$ and $v_{\mathrm{Q}}$ to account for the strong inhomogeneity of QI over the QD 
(see the Methods section). The result of this full simulation is shown in Fig. 3d. We highlight that the good qualitative agreement with our experimental results (Fig. 2a) is rather insensitive to the set of parameters used in our simulation. In particular, the choice of the distribution for the parameters $\theta$ and $v_{\mathrm{Q}}$ did not affect our results significantly. Furthermore, our simulation treats the QD spin system as a pure spin-3/2 system, whereas for In $I=9 / 2$. A numerical treatment of the full InGaAs nuclearspin system is beyond the scope of this paper and would most probably not alter the qualitative behaviour of our simulations (see the Methods section).

Our results show that the nuclear-spin system of a self-assembled QD provides a rare example for a solid-state nuclear-spin ensemble that cannot be described by a nuclear-spin temperature ${ }^{23}$. We note that, if we could assign a spin temperature to the QD nuclear-spin system, optical pumping combined with adiabatic demagnetization of the nuclear spins would be a novel and efficient means of nuclear-spin cooling in QDs without QI: possible systems include nuclear spin-1/2 systems, such as ${ }^{13} \mathrm{C}$-nanotube QDs (ref. 24), where QI is inherently absent, or strain-free semiconductor nanostructures ${ }^{25}$, such as epitaxially grown droplet QDs (ref. 26). There, adiabatic nuclear-spin cooling would be limited only by nuclear dipolar interactions resulting in $B_{\mathrm{loc}} \approx 0.1 \mathrm{mT}$. Achieving nuclear-spin cooling to temperatures of $\approx 100 \mathrm{nK}$ should be feasible in these systems, opening ways to study the remnants of nuclear magnetic phase transitions in the mesoscopic system of QD nuclear spins $^{27}$.

\section{Methods}

Sample and experimental techniques. Individual QDs were studied using the photoluminescence of $X^{-1}$ under resonant excitation of an excited QD state. The QD sample was grown by molecular-beam epitaxy on a (100) semi-insulating GaA substrate. The approximate composition of the QDs after self-assembled growth and postgrowth annealing was $\operatorname{In}_{0.5} \mathrm{Ga}_{0.5}$ As. For individual optical addressing, the QDs were grown at a low density of $\lesssim 0.1 \mu^{-2}$. The QDs were spaced by $25 \mathrm{~nm}$ of GaAs from a doped $\mathrm{n}^{2+}$-GaAs layer, followed by $30 \mathrm{~nm}$ of GaAs and 29 periods of an AlAs/GaAs (2/2 nm) barrier, which was capped by $4 \mathrm{~nm}$ of GaAs. A bias voltage applied between the top Schottky and back Ohmic contacts controls the charging state of the QD. Optical pumping of QD nuclear spins was performed at the centre of the $X^{-1}$ stability plateau in gate voltage, where photoluminescence counts as well as the resulting Overhauser shift were maximized ${ }^{13}$.

The QD sample was immersed in a liquid helium bath cryostat equipped with a superconducting magnet and was held at the cryostat base temperature of $1.7 \mathrm{~K}$. The photoluminescence emitted by the QD was analysed in a $750 \mathrm{~mm}$ monochromator, allowing for the determination of spectral shifts of the QD emission lines with a precision of $\sim 1 \mu \mathrm{eV}$ (ref. 12). A combination of an optica 'pump-probe' technique, together with linear ramps of the applied magnetic field, was used to adiabatically demagnetize the QD nuclear spins (see Fig. 1c); technical details of the pump-probe set-up are given elsewhere'. The 'pump' pulse consists of a circularly polarized laser pulse of duration $\tau_{\text {pump }}$, which is used to optically orient the QD nuclear spins ${ }^{12}$. We typically achieve an Overhauser shift of $\Delta E_{\mathrm{OS}}=60 \mu \mathrm{eV}$ at $B_{\mathrm{i}}=1 \mathrm{~T}$, corresponding to nuclear-spin polarization $P_{\text {nuc }} \approx 35 \%$ or $T_{\mathrm{i}} \approx 1.5 \mathrm{mK}$ (for $B_{\mathrm{i}}=2.2 \mathrm{~T}, \Delta E_{\mathrm{OS}}=89.5 \mu \mathrm{eV}$ and $P_{\text {nuc }} \approx 50 \%$ ). In the range of $B_{\text {ext }}$ relevant to our experiment, $P_{\text {nuc }} \propto B_{\mathrm{i}}$ (ref. 12) such that the initial nuclear-spin temperature $T_{\mathrm{i}}$ is roughly constant and of the order of few millikelvin (ref. 6) for all values of $B_{\text {i }}$

Directly after applying the pump pulse to the QD, the gate voltage is switched to a value where the QD is charge neutral. In this regime, nuclear-spin polarization has an exceedingly long relaxation time of the order of hours ${ }^{9}$ (see Fig. la). We note that we can exclude any significant nuclear polarization of the bulk material surrounding the QD. The observation of dynamical nuclear-spin polarization in our experiment depends sensitively on the excitation laser energy, which we tune to an intra-dot ( $p$-shell) excitation resonance with a width of $\approx 300 \mu \mathrm{eV}$ and located $\approx 36 \mathrm{meV}$ above the photoluminescence emission energy. The sharpness and energy of this excitation resonance makes any excitation processes that involve the creation of free electrons in the bulk very unlikely ${ }^{28}$. Furthermore, the pumping time $\tau_{\text {pump }}=600 \mathrm{~ms}$ used in our experiment is much too short to lead to a significant bulk nuclear-spin polarization, even if some free electrons were created during laser illumination.

Details of the model. The model we developed to explain our experimental findings is based on the steady-state solution of a rate equation for the populations of a nuclear-spin $I=3 / 2$ system. The nuclear spins are initialized with a Boltzmann distribution over the spin states at $B_{\text {ext }}=B_{\mathrm{i}}$. The assumption of a thermal distribution of nuclear-spin levels at $B_{\text {ext }}=B_{\mathrm{i}}$ is justified by the fact that nuclear spins are polarized by hyperfine interaction with the QD electron: optical pumping of the electron leads to a broadening of its spin states by several $\mu \mathrm{eV}$ (ref. 12), allowing for electron-nuclear flip-flops between the electron and any two given nuclear-spin states which are coupled by the hyperfine interaction. It is therefore reasonable to assume that the occupations of nuclear-spin levels at $B_{\mathrm{i}}$ follow a Boltzmann distribution.

We then change the magnetic field by keeping the populations of spin levels fixed. Only at the specific fields where cross-relaxation is permitted (Fig. 3c) do we allow for a local thermal equilibrium to be established between the spin levels involved in the cross-relaxation transitions. All other populations and the total energy of the nuclear-spin system remain constant. On sweeping through $B_{\text {ext }}=0$, we assume that the levels $m_{z^{\prime}}= \pm 1 / 2$ undergo an adiabatic passage through an anticrossing induced by the coupling of these two states by dipolar interactions. Spin states with $m_{z^{\prime}}= \pm 3 / 2$ however remain uncoupled and undergo an adiabatic level crossing, which preserves their populations.

The result of our simulations is shown in Fig. 3c,d. We illustrate the evolution of the occupations of the individual nuclear-spin states in Fig. $3 \mathrm{c}$, where we show the spectrum of a nuclear spin for the parameters $v_{\mathrm{Q}}=3 \mathrm{MHz}, \theta=0.3 \pi / 2$ and $\gamma=10 \mathrm{MHz} \mathrm{T}^{-1}$. The occupations of the individual levels are encoded by the thickness and grey shade of the corresponding lines. Magnetic fields where cross-relaxation processes take place are indicated by red lines. We repeated this calculation for a set of angles $\theta \in\{0.1,0.2, \ldots, 0.6\} \frac{\pi}{2}$ and quadrupolar frequencies $v_{\mathrm{Q}} \in\{-4,-3,-2,2,3,4\} \mathrm{MHz}$, over which we average our results. As the local strain in our QDs can be both tensile and compressive, positive and negative values for $v_{\mathrm{Q}}$ are possible. By solving the complete Hamiltonian $\hat{H}_{\mathrm{Q}}+\hat{H}_{\mathrm{Z}}$, we can relate the occupancies of the spin levels to our experimentally observed nuclear-spin polarization-the expectation value $\left\langle\hat{I}_{z}\right\rangle$ of the nuclear-spin polarization along the direction of $B_{\text {ext }}$. Figure $3 \mathrm{~d}$ of the main paper shows the result of our simulation in the form of the calculated evolution of $P_{\text {nuc }}$ as a function of $B_{\mathrm{f}}$.

We note that our model is a great simplification of the actual experimental situation. First, we completely ignore cross-relaxation events between nuclei of different $\left(\theta, v_{\mathrm{Q}}\right)$ - values. Second, our calculation was performed for a spin-3/2 system for simplicity, whereas the actual QD nuclear-spin system consists of a mixture of spin $3 / 2(\mathrm{Ga}, \mathrm{As})$ and spin 9/2 (In), which further complicates the situation. Although a numerical treatment of the full InGaAs nuclear-spin system is beyond the scope of this paper, we argue that such a treatment would not alter the physical picture conveyed by our simulation. Including $I=9 / 2$ spins would lead to a nuclear-spin spectrum similar to the one illustrated in Fig. $3 \mathrm{~b}$. The number of magnetic-field values where cross-relaxation events would be energetically allowed would increase compared with the case of $I=3 / 2$, but these events would still be singular in the sense that for most values of $B_{\text {ext }}$ the nuclear spins could not thermalize. The system would thus still be driven out of thermal equilibrium and the relaxation events during cross-relaxation would lead to an increase of nuclear-spin entropy. Including flip-flop events between In and As nuclear spins would have a similar effect: these transitions would be allowed for a subset of close nuclei and would allow for partial thermalization only at specific values of $B_{\text {ext }}$.

Received 16 January 2009; accepted 3 April 2009; published online 10 May 2009

\section{References}

1. Goldman, M. Spin Temperature and Nuclear Magnetic Resonance in Solids (Oxford Univ. Press, 1970).

2. Abragam, A. \& Proctor, W. G. Experiments on spin temperature. Phys. Rev. 106, 160-161 (1957).

3. Slichter, C. P., Holton, W. C. \& Fellow, A. P. S. Adiabatic demagnetization in a rotating reference system. Phys. Rev. 122, 1701-1708 (1961).

4. Purcell, E. M. \& Pound, R. V. A nuclear spin system at negative temperature Phys. Rev. 81, 279-280 (1951).

5. Meier, F. Optical Orientation (North-Holland, 1984)

6. Dyakonov, M. I. \& Perel, V. I. Optical orientation in a system of electrons and lattice nuclei in semiconductors-theory. Sov. Phys. JETP 38, 177-183 (1974).

7. Paget, D. Optical-detection of NMR in high-purity GaAs-direct study of the relaxation of nuclei close to shallow donors. Phys. Rev. B 25, 4444-4451 (1982).

8. Kalevich, V. K., Kul'kov, V. D. \& Fleisher, V. G. Onset of a nuclear polarization front due to optical spin orientation in a semiconductor. JETP Lett. 35, 20-24 (1982).

9. Maletinsky, P., Badolato, A. \& Imamoglu, A. Dynamics of quantum dot nuclear spin polarization controlled by a single electron. Phys. Rev. Lett. 99, 056804 (2007).

10. Gammon, D. et al. Nuclear spectroscopy in single quantum dots: Nanoscopic Raman scattering and nuclear magnetic resonance. Science 277, 85-88 (1997)

11. Eble, B. et al. Dynamic nuclear polarization of a single charge-tunable InAs/GaAs quantum dot. Phys. Rev. B 74, 081306 (2006).

12. Maletinsky, P., Lai, C. W., Badolato, A. \& Imamoglu, A. Nonlinear dynamics of quantum dot nuclear spins. Phys. Rev. B 75, 035409 (2007). 
13. Lai, C. W., Maletinsky, P., Badolato, A. \& Imamoglu, A. Knight-field-enabled nuclear spin polarization in single quantum dots. Phys. Rev. Lett. 96, 167403 (2006).

14. Overhauser, A. W. Polarization of nuclei in metals. Phys. Rev. 92, 411-415 (1953).

15. Malinowski, A., Brand, M. A. \& Harley, R. T. Nuclear effects in ultrafast quantum-well spin-dynamics. Physica E 10, 13-16 (2001).

16. Braun, P.-F., Urbaszek, B., Amand, T. \& Marie, X. Bistability of the nuclear polarization created through optical pumping in $\mathrm{In}_{1-x} \mathrm{Ga}_{x}$ As quantum dots. Phys. Rev. B 74, 245306 (2006).

17. Dzhioev, R. I. \& Korenev, V. L. Stabilization of the electron-nuclear spin orientation in quantum dots by the nuclear quadrupole interaction. Phys. Rev. Lett. 99, 037401 (2007).

18. Maletinsky, P. Polarization and Manipulation of a Mesoscopic Nuclear Spin Ensemble Using a Single Confined Electron Spin. PhD thesis, ETH Zürich (2008).

19. Deng, C. X. \& Hu, X. D. Selective dynamic nuclear spin polarization in a spin-blocked double dot. Phys. Rev. B 71, 033307 (2005).

20. Slichter, C. P. Principles of Magnetic Resonance (Springer, 1996).

21. Williamson, A. J. \& Zunger, A. InAs quantum dots: Predicted electronic structure of free-standing versus GaAs-embedded structures. Phys. Rev. B 59, 15819-15824 (1999).

22. Sundfors, R. K., Tsui, R. K. \& Schwab, C. Experimental gradient elastic tensors: Measurement in I-VII semiconductors and the ionic contribution in III-V and I-VII compounds. Phys. Rev. B 13, 4504-4508 (1976).
23. Rhim, W.-K., Pines, A. \& Waugh, J. S. Violation of the spin-temperature hypothesis. Phys. Rev. Lett. 25, 218-220 (1970).

24. Churchill, H. O. H. et al. Electron-nuclear interaction in ${ }^{13} \mathrm{C}$ nanotube double quantum. Nature Phys. 5, doi:10.1038/NPHYS1247 (2008).

25. Feng, D. H., Akimov, I. A. \& Henneberger, F. Nonequilibrium nuclear-electron spin dynamics in semiconductor quantum dots. Phys. Rev. Lett. 99, 036604 (2007).

26. Belhadj, T. et al. Optically monitored nuclear spin dynamics in individual GaAs quantum dots grown by droplet epitaxy. Phys. Rev. B 78, 205325 (2008).

27. Simon, P. \& Loss, D. Nuclear spin ferromagnetic phase transition in an interacting two dimensional electron gas. Phys. Rev. Lett. 98, 156401 (2007).

28. Vasanelli, A., Ferreira, R. \& Bastard, G. Continuous absorption background and decoherence in quantum dots. Phys. Rev. Lett. 89, 216804 (2002).

\section{Acknowledgements}

We thank A. Högele, J. Elzerman and S. D. Huber for help with the manuscript, and T. Amand and O. Krebs for discussions. We acknowledge A. Badolato for sample growth. This work is supported by NCCR-Nanoscience and an ERC Advanced Investigator Grant.

\section{Additional information}

Reprints and permissions information is available online at http://npg.nature.com/ reprintsandpermissions. Correspondence and requests for materials should be addressed to P.M. or A.I. 